\title{
The difficult management of systemic-onset juvenile rheumatoid arthritis. Level of serum ferritin as aspecific diagnostic finding ${ }^{*}$
}

\author{
Maria Giovanna Colella ${ }^{1 \#}$, Giuseppe Buttaro ${ }^{1}$, Lucia Masi ${ }^{1}$, Elena Palma ${ }^{1}$, Raffaele Amelio ${ }^{1}$, \\ Alexander Vallone ${ }^{2}$ \\ ${ }^{1}$ Pediatric Unit, “Dono Svizzero” Hospital, Formia, Italy \\ ${ }^{2}$ Faculty of Medicine and Surgery, Università Cattolica, Rome, Italy \\ Email: ${ }^{\text {mggcolella@hotmail.it }}$
}

Received 20 July 2011; revised 4 December 2011; accepted 23 December 2011

\begin{abstract}
Systemic-onset JRA is characterized by spiking fevers lasting more than two weeks, typically occurring once, twice each day, with temperature returning to the normal or below normal. There are no specific diagnostic tests for systemic JRA. High level of serum ferritin is the most important, no specific, diagnostic finding associated.
\end{abstract}

Keywords: Fever of Unknown Origin; Juvenile Rheumatoid Arthritis; Ferritin

\section{INTRODUCTION}

Juvenile idiopathic arthritis (JIA) is the term used to describe a group of inflammatory joint diseases of unknown etiology, with onset before the age of 16, with a duration longer than 6 weeks [1,2]. The definition of JIA has been chosen to indicate the absence of a known mechanism responsible for the disease and to stress the need to exclude other forms of arthritis that occur in association with other diseases (especially arthritis is associated with diseases hematology-oncology, inflammatory and infectious diseases). Systemic-onset juvenile rheumatoid arthritis representing about $10 \%$ of JIA is characterized by spiking fevers lasting more than two weeks, typically occurring once or twice each day, at about the same time of day, with temperature returning to the normal or below normal $[3,4]$ The fever pattern is very useful because infections, Kawasaki desease, and malignancy do not have such a predictable pattern [5].

We present two cases of systemic juvenile rheumatoid arthritis at the onset that presented fever of unknown origin, characterized by arthralgias migrants, evanescent

\footnotetext{
${ }^{*}$ Conflict of interest declarations.

The authors have no conflicts of interest relevant to this article to disclose. ${ }^{\#}$ Corresponding author.
}

rash and splenomegaly. The diagnosis was suggested by these findings in association with a double quotidian fever and a highly elevated serum ferritin level.

\section{CASE 1}

Child of 12 years old came to our observation for five days of persistent high fever, muscle pain, leg weakness and difficulty in walking which worsens with increasing temperature.

Family and past personal history were not contributory. In the month before the hospitalization, the parents referred complaining pain in the right temporo mandibular joint and than, after two weeks, deficit in walking on the morning after the use of rotating carpet. This problem was resolved with a single dose of paracetamol. In the following week he presented diarrhea for two days, slight increase in temperature and wearying. The parents also reported use of home-made eggs to eat and contact with family pets in occasion of visits to his grandmother who lived in the countryside.

At admission, physical examination was not contributory. But because he showed signs of leg pain and back pain, a lumbar puncture ruled out problems with meningeal involvement.

During hospitalization, his medical history showed intermittent fever, with at least two episodes a day for eighteen days, spine arthralgia (especially cervical and lumbosacral) and hip joint articulation, associated with functional impotence of the lower limbs, especially in the morning and during the peak of the fever; the tendon reflexes were symmetric and vivid, always. On three occasions, a pink salmon rash appeared on the neck, trunk and upper limbs, in association with fever (Figure 1). No improvement in clinical and laboratory response to broad-spectrum antibiotic therapy given for ten days he showed leukocytosis, persistently inflammatory markers (CRP, ESR) elevated, mild anemia, modest ele- 


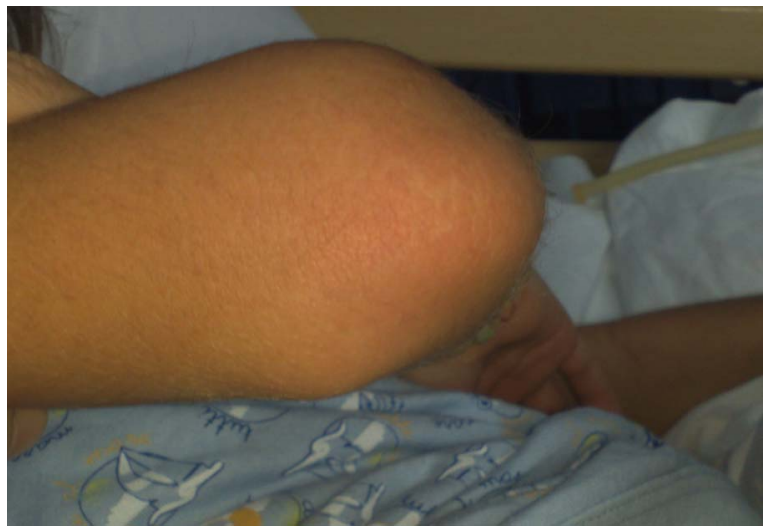

Figure 1. Salmon-pink rash on the limb.

vation of transaminases and lactate dehydrogenase, normocomplementemia; serum ferritin level increased gradually from value of $1389 \mathrm{ng} / \mathrm{ml}$ to 12.000 (Table 1). An infectious workup was negative, including stool ova and parasites.

Antibodies for common autoimmune diseases were in the norm repeated twice. General coltural tests and blood coltures were negative, repeated several times.

A peripheral blood smear and then a bone marrow excluded malignancies; lymphocyte subpopulations were normal. Fundus examination was normal. An echo handwriting abdomen, followed by a CAT scan chest, abdomen and skull showed splenomegaly (splenic diameter: $14.5 \mathrm{~cm}$ ). Magnetic resonance imaging of the brain excluded an issue of acute inflammatory neurologic disease. No coronaropathy and valvulopathy reperted.

The persistence of high values of inflammatory markers, in association with further raising of serum ferritin levels and the fever profile diagnosed of systemic onset JRA. Arthritis was not present yet, but there were diffuse arthralgias and with time the child was not able to use his thumb to play games. A complicating MAS has considered and then excluded [5,6].

Beacause child was not responsive to ibuprofen therapy, after a second negative bone marrow examination, he started prednisone treatment with significant improvement of clinical conditions.

\section{CASE 2}

Girl of 9 years was under our observation for persistent fever for five days and asthenia unresponsive to antibiotic and occasionally treatment with bethametasone. At admission, general conditions were expired; she presented a widespread multiforme erythema, right ankle swelling and edema of small phalanges of the hands (Figure 2). A diffuse arthralgia above the upper limbs and column was reported.

Personal history was not contributive. The father was affected from vitiligo; the mother had an autoimmune
Table 1. Values of white blood cells, inflammatory markers and serum ferritin in relation to days of hospitalization-Case 1.

\begin{tabular}{cccccc}
\hline & $\mathbf{1 4 / 1 0 / 1 0}$ & $\mathbf{1 9 / 1 0 / 1 0}$ & $\mathbf{2 5 / 1 0 / 1 0}$ & $\mathbf{2 9 / 1 0 / 1 0}$ & $\mathbf{0 2 / 1 1 / 1 0}$ \\
\hline WBC $10^{3} / \mu \mathrm{l}$ & 13870 & 30490 & 31330 & 25880 & 17250 \\
$\mathrm{ESR} \mathrm{mm} / \mathrm{hr}$ & 70 & 108 & 104 & 105 & 80 \\
$\mathrm{CRP} \mathrm{mg} / \mathrm{dl}$ & 13.58 & 15.45 & 8.71 & 13.76 & 6.32 \\
Ferritin ng/ml & 1 & 3066 & 4360 & 12461 & 8263 \\
$\begin{array}{c}\text { D-Dimers mg/l } \\
\text { (v.n. 0.60mg/l) }\end{array}$ & & & 7.55 & 5.14 & \\
\hline
\end{tabular}

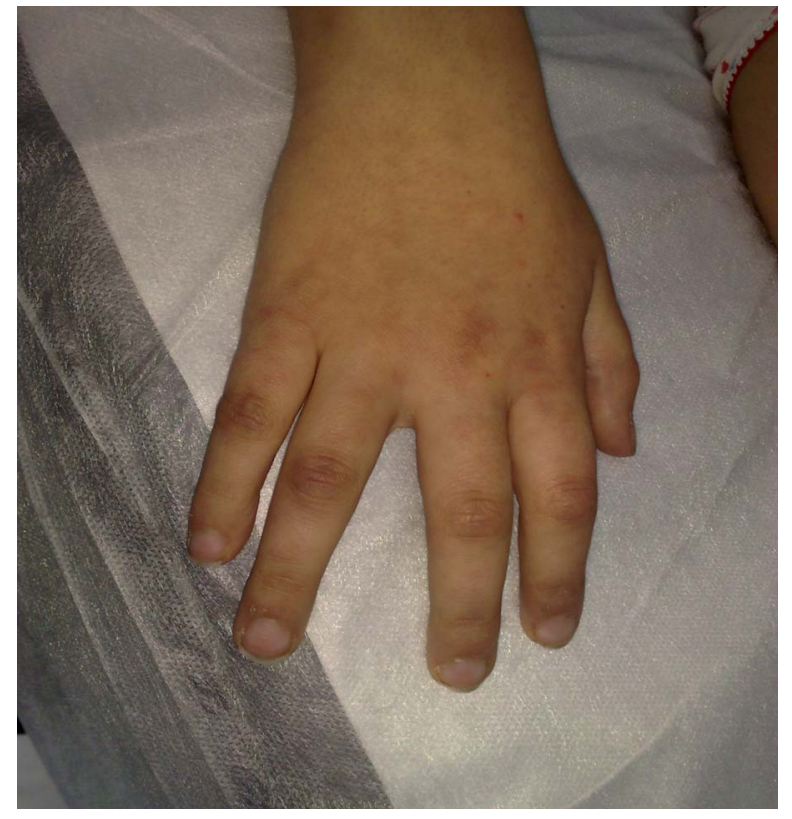

Figure 2. Multiforme erythema and edema of small phalanges of the hands.

tyroiditis. Her brother was suffering from allergic asthma.

Laboratory tests were characterized by elevated erythrocyte sedimentation rate, high level of serum ferritin and positive C-reactive protein (Table 2).

An infectious workup was negative. Antibodies for common autoimmune were in the norm. The echocardiogram, chest radiograph were normal. Abdominal ultrasound showed mild splenomegaly. An examination of the fundus was normal. General coltural tests and blood coltures, peripheral blood smear were negative. Serum ferritin level increased gradually to value of $5881 \mathrm{ng} / \mathrm{ml}$.

The fever was intermittent at least two daily peaks; the rash seemed to coincide with the rise in body temperature. The course of the fever, the association with arthralgia, ankle edema and erythema and phalanges with high value of ferritin.suggested systemic-onset JRA. After a sample of bone marrow to rule out malignancy, it has been initiated treatment with prednisone with remission of symptoms and levels of ferritin .The therapy with ibuprofen was inefficay. 
Table 2. Values of white blood cells, inflammatory markers and serum ferritin in relation to days of hospitalization before and after beginning of cortisonic terapy-Case 2 .

\begin{tabular}{|c|c|c|c|c|c|}
\hline & $26 / 04 / 11$ & $28 / 04 / 11$ & $\begin{array}{c}\text { Beginning } \\
\text { of therapy } \\
02 / 05 / 11\end{array}$ & $\begin{array}{c}\text { After } \\
\text { therapy } \\
\text { 07/05/11 }\end{array}$ & $\begin{array}{c}\text { After } \\
\text { therapy } \\
13 / 05 / 11\end{array}$ \\
\hline WBC $10^{3} / \mu \mathrm{l}$ & 10.060 & 15.840 & & 7.520 & 9678 \\
\hline ESR mm/hr & 65 & 45 & & 49 & 18 \\
\hline CRP mg/dl & 6.4 & 5.75 & & 2.23 & 0.18 \\
\hline Ferritin ng/ml & 4723 & 5881 & & 934 & 267 \\
\hline $\begin{array}{l}\text { D-Dimers mg/l } \\
\text { v.n. } 0.60 \mathrm{mg} / \mathrm{l}\end{array}$ & 2.03 & 4.15 & & 0.58 & 0.44 \\
\hline
\end{tabular}

\section{DISCUSSION}

There are no specific diagnostic tests for sistemic JRA, which commonly presents as prolonged fever that is not easily diagnosed (i.e., FUO) [6]. Typically, patients with JRA present with liver/spleen involvement, pauci-articular arthritis, arthralgias and myalgias,ocular involvement, and evanescent salmon-colored truncal rash. Arthralgia occurs in other disorders [6,7]. Serum ferritin levels may be elevated in infections, malignancies (leukaemia, lymphomas), liver diseases and haemochromatosis. However, in these conditions serum ferritin concentrations rarely exceed values of $>3000 \mathrm{ng} / \mathrm{ml}$. High level of serum ferritin is the most important, non specific, diagnostic finding associated with JRA [8-10]. Clinicians should appreciate the diagnostic significance of fever patterns and the diagnostic significance of elevated serum ferritin levels in patients with FUO.

\section{REFERENCES}

[1] Petty, R.E., Southwood, T.R., Manners, P., et al. (2004) International league of associations for rheumatology classification of juvenile idiopathic arthritis: Second revision, Edmonton, 2001. Journal of Rheumatology, 31, 390-392.

[2] Berntson, L., Andersson Gäre, B., Fasth, A., et al. (2003) Incidence of juvenile idiopathic arthritis in the Nordic countries. A population based study with special reference to the validity of the ILAR and EULAR criteria. Nordic Study Group. Journal of Rheumatology, 30, 22752282.

[3] Riise, Ø.R., Handeland, K.S., Cvancarova, M., et al. (2008) Incidence and characteristics of arthritis in Norwegian children: A population-based study. Pediatrics, 121, e299-e306. doi:10.1542/peds.2007-0291

[4] Woo, P. (2006) Systemic juvenile idiopathic arthritis: Diagnosis, management and outcome. Nature Reviews Rheumatology, 2, 28-34. doi:10.1038/ncprheum0084

[5] Ravelli, A., Magni-Manzoni, S., Pistorio, A., et al. (2005) Preliminary diagnostic guidelines for macrophage activation syndrome complicating systemic juvenile idiopathic arthritis. Journal of Pediatrics, 146, 598-604. doi:10.1016/j.jpeds.2004.12.016

[6] Insalaco, A. (2006) Macrophage activation syndrome in juvenile idiopathic arthritis. Acta Paediatrica, 95, 38-41.

[7] Lomater, C., Gerloni, V., Gattinara, M., et al. (2000) Systemic onset juvenile idiopathic arthritis: A retrospective study of 80 consecutive patients followed for 10 years. Journal of Rheumatology, 27, 491-496.

[8] Yao, T.C., Kuo, M.L., See, L.C., Ou, L.S., Lee, W.I., Chan, C.K. and Huang, J.L. (2006) RANTES and monocyte chemoattractant protein 1 as sensitive markers of disease activity in patients with juvenile arthritis: A sixyear longitudinal study. Arthritis \& Rheumatism, 54, 25852593.

[9] Meijvis, H., Endeman, A.B.M., Geers, E.J. and Borg, E.J. (2007) Extremely high serum ferritin levels as diagnostic tool in adult-onset still's disease. The Netherland Journal of Medicine, 65, 212-214.

[10] Jandus, P., Wang, W., Seitz, M., et al. (2010) High serum ferritin in adult-onset still's disease. International Journal of Clinical Medicine, 1, 81-83.

\author{
ABBREVIATIONS USED \\ JRA: Juvenile Rheumatoid Arthritis \\ CRP: C-Reactive Protein \\ ESR: Erythro-Sedimentation-Rate \\ CAT: Computed Axial Tomography \\ MAS: Macrophage Activation Syndrome \\ FUO: Fever of Unknown Origin
}

